\title{
Controlled Release of Acetyl Salicylic Acid in Intelligent Hydrogel Based on Cross-linked 0- Carboxymethyl Chitosan
}

Yiguang Wu

Shenzhen University

Muhammad Shahid Riaz Rajoka

Shenzhen University

Jun Zeng

Shenzhen University

Hafiza Mahreen Mehwish

Shenzhen University

Wenli Liu

Shenzhen University

Liqing Zhao ( $\square$ lqzhao@szu.edu.cn )

Shenzhen University

\section{Research Article}

Keywords: 0-carboxymethyl chitosan, acetylsalicylic acid, hydrogel, controlled drug release, pH sensitivity

Posted Date: March 18th, 2021

DOl: https://doi.org/10.21203/rs.3.rs-312464/v1

License: (1) This work is licensed under a Creative Commons Attribution 4.0 International License.

Read Full License 


\section{Correspondence author}

12 Professor Liqing Zhao

13 Tel: +86-755-26550395

14 Fax: +86-755-26733095

15 E-mail: lqzhao@szu.edu.cn

16 \# These authors contributed equally

17 Author's Email IDs

18 Yiguang Wu (yebsd@sina.com)

19 Muhammad Shahid Riaz Rajoka (shahidrajoka@yahoo.com)

20 Jun Zeng (2172226008@email.szu.edu.cn)

21 Hafiza Mahreen Mehwish (mahreen.mehwish@yahoo.com)

22 Xia Lixin (xialixin@126.com)

23 Wenli Liu (61950096@qq.com)

24 Liqing Zhao (lqzhao@szu.edu.cn) 


\section{Abstract}

Drug-loading hydrogels were prepared from O-carboxymethyl chitosan (O-CMCS) with a high degree of substitution and different deacetylation degree (DD) using acetylsalicylic acid as drug and glutaraldehyde as a cross-linking agent. Also, the DD's effect on the controlled drug release performance of drug-loading particle was explored. The results showed that the hydrogels and particles were prepared from $5 \mathrm{~g}$ of $\mathrm{O}-$ CMCS solution (4.0 wt.\%), $2.5 \mathrm{~g}$ of sodium acetylsalicylate solution (8.0 wt.\%), and $1.5 \mathrm{~mL}$ of glutaraldehyde solution (1.0 wt.\%). The interior pore size of the particle ( $\mathrm{DD}=51 \%)$ was between 50 and $100 \mu \mathrm{m}$, and its cumulative drug release ratios in the simulated gastric and intestinal juices were of the top values. The drug release of the drug-loading particle was proved to be obviously $\mathrm{pH}$ sensitive and it can be applied as a colonic drug.

Keywords: O-carboxymethyl chitosan; acetylsalicylic acid; hydrogel; controlled drug release; $\mathrm{pH}$ sensitivity

\section{Introduction}

Besides the discovery of the huge number of therapeutic agents, few have shown the therapeutic success after systematic administration because of their low bioavailability[1]. Nowadays, numerous approaches including the in situ gelling system have been developed to improve the bioavailability of therapeutic agents [2]. In these in situ gelling systems, the therapeutic agents such as drugs can be encapsulated into the matrix which reduced the unexpected side effect associated with systematic administration [3]. The early development of these hydrogels system mainly focuses on the use of synthetic materials such as copolymers of $N$ isopropylacrylamide and poly(ethylene glycol)/poly(lactic acid) block copolymers [4]. These materials were suitable for local drug delivery, but on the other hand, the non-biodegradability nature of these materials limits their clinical applications [5]. Therefore, recently the focus has been oriented towards naturally polymers having biodegradability and biocompatibility properties that can also form easily hydrogels [5-6]. Among natural polymers, the chitosan obtained from chitin has gained considerable attention in the drug delivery system due to its biodegradability and biocompatibility nature [7].

A recent study showed that the chitosan and its derivative can be used as a carrier of drug delivery system with no reported toxicity along with excellent biocompatibility and biodegradability[8]. When chitosan was modified by carboxymethylation and deacetylation, the corresponding derivatives carboxymethyl chitin/chitosan (CMCH/CMCS) were found to be water soluble and showed excellent biocompatibility of an amphoteric polymer [9]. The $\mathrm{CMCH}$ had been used as tablet additives by Huang et 
al. (2012), and the CMCS is currently being investigated in this work as a drug carrier. Theoretically, in the acidic conditions, CMCS with a high degree of substitution (DS) shows many carboxyl (-COOH) groups. However, in the neutral or alkaline condition, these - $\mathrm{COOH}$ groups can be transformed into COO-. Consequently, CMCS itself shall be $\mathrm{pH}$ sensitive. Interestingly, the molecular chain of CMCS contains certain amounts of $-\mathrm{NH} 2$, which can react with glutaraldehyde to form the Schiff Base. Therefore, it's hopeful to prepare the drug-loading hydrogel by cross-linking the drug-containing CMCS solution with glutaraldehyde in order to control the release of interior drug by its contraction and expansion due to the carboxyl's conversion under different $\mathrm{pH}$ environments [10]. Hence, hydrogels themselves are promising biomaterials with basic ability to control the transportation of nutrients, $\mathrm{O} 2$, and other water-soluble metabolites through diffusion. They also have the capability of many other desirable physicochemical indications and of being a cell growth substrate.

The acetylsalicylic acid (aspirin) is non-steroidal drugs having anti-inflammatory, analgesic, and antipyretic effects [11]. Furthermore, the oral intake of a high dose of acetylsalicylic acid may have undesirable side effects including tinnitus, gastric bleeding, and ulcers [12]. Therefore, it would be better if the function of control release of acetylsalicylic acid combines on to the acetylsalicylic acid containing drugs so that the acetylsalicylic acid would be gradually released to form a proper drug concentration in the plasma. Therefore, the combination of chitosan as a drug carrier along with the acetylsalicylic acid drug would be an excellent candidate for the polymeric drug for development of controlled release of acetylsalicylic acid.

In this paper, a series of O-carboxymethyl chitosan (O-CMCS) with a high DS and different deacetylation degrees (DD) were prepared from the primary raw material, chitin. The corresponding hydrogels were prepared by cross-linking O-CMCS solution containing acetylsalicylic acid with glutaraldehyde, while the drug-loading particles were prepared by drying the drug-loading hydrogels. Also, the controlled drug release performance of these drug-loading particles was carefully examined.

\section{Experimental}

\section{Preparation and structural parameters' determination of series of $O$-CMCS}

A two-step method was used to prepare a series of O-CMCS with a high DS and different DD. In the first step, O-carboxymethyl chitin (O-CMCH) with a high DS and a low DD was developed. This reaction was made under the optimum conditions including the molar ratio (chitin unit to chloroacetic acid to $\mathrm{NaOH}$ ), the concentration of $\mathrm{NaOH}$, the reaction temperature, and the reaction time as outlined elsewhere[13]. In the second step, the series of O-CMCS with a high DS and different DD were obtained. Here, the concentration of $\mathrm{NaOH}$, the reaction temperature and the reaction time were all controlled.

$200 \mathrm{~g}$ of chitin powder was added into $1,300 \mathrm{~mL}$ of $\mathrm{NaOH}$ solution $(23 \mathrm{wt} . \%)$ with stirring, and the slurry was vacuumized overnight by a water pump to form alkalized chitin. As a dispersant, an 
appropriate amount of isopropyl alcohol was added into the alkalized chitin, and the suspension was stirred at $60^{\circ} \mathrm{C}$. Then, isopropyl alcohol solution containing $326 \mathrm{~g}$ of chloroacetic acid was added; allowed to react for $4 \mathrm{~h}$, and filtered. The filtered residue was dissolved in pure water and neutralized with concentrated $\mathrm{HCl}$ solution. Ultrafiltration membrane with a molecular weight retention value of 10,000 was used to ultrafiltrate 5 times where the volume compression ratio was $1 / 5$ each time. Finally, the concentrated solution was dried in an oven at $80^{\circ} \mathrm{C}$ and then placed in a vacuum oven at $60^{\circ} \mathrm{C}$ overnight to acquire $20.24 \mathrm{~g}$ of $\mathrm{O}-\mathrm{CMCH}, \mathrm{F} 1$.

Furthermore, $2 \mathrm{~g}$ of $\mathrm{F} 1$ was added to $70 \mathrm{~mL}$ of concentrated $\mathrm{NaOH}$ solution to dissolve it entirely. After deacetylation reaction at a certain temperature for a definite time, the solution was neutralized with concentrated $\mathrm{HCl}$ solution. Subsequently, ultrafiltration membrane with a molecular weight retention value of 10,000 was used again for 5 times' ultrafiltration. As usual, in each of these times, the volume compression ratio was $1 / 5$. Finally, the concentrated solution was dried in an oven at $80^{\circ} \mathrm{C}$ and placed in a vacuum oven overnight at $60^{\circ} \mathrm{C}$ to obtain $\mathrm{O}-\mathrm{CMCS}, \mathrm{F} 2 \sim \mathrm{F} 5$. Respective reaction conditions $(\mathrm{NaOH}$ concentration, reaction temperature and time) of F2 F5 were given in Table 1.

Besides, $0.2 \mathrm{~g}$ of each O-CMCS sample was added into $50 \mathrm{~mL}$ of $\mathrm{NaCl}$ solution $(0.1 \mathrm{~mol} / \mathrm{L})$ in a conical flask and then stirred until completely dissolved. An aliquant amount of $\mathrm{HCl}$ solution $(0.1 \mathrm{~mol} / \mathrm{L})$ was used to adjust the $\mathrm{pH}$ value to 3.0, and after that, the solution was titrated with a $0.05 \mathrm{~mol} / \mathrm{L}$ of standard $\mathrm{NaOH}$ solution. Its $\mathrm{pH}$ value was verified by a $\mathrm{pH}$ meter (Orion 720A, Thermo Scientific, USA) with an interval of 0.04 , and the consumption volume $(\mathrm{V}, \mathrm{mL})$ of the $\mathrm{NaOH}$ solution was recorded. The experimental data were analyzed through a differential titration curve, where: $\triangle \mathrm{pH} / \triangle \mathrm{V}$ and $\mathrm{V}(\mathrm{mL})$ was earmarked as the ordinate and abscissa, respectively. And the peak $\mathrm{pH}$ values for $-\mathrm{COOH}$ and $-\mathrm{NH} 2$ were 5.5 and 9.6 , respectively.

The contents of $\mathrm{C}$ and $\mathrm{N}$ elements in these O-CMCS samples were measured by an Elemental Analyzer (Vario EL III, Elementary, Germany) with high-temperature combustion and complete decomposition methods. For interaction relationships among the DD, the DS, the content ratio of $\mathrm{C}$ and $\mathrm{N}$ elements, and the titration parameters, Eqs. (1) and (2) were framed.

$$
C / N=\frac{[6+(1-D D) \times 2+D S \times 2] \times 12}{1 \times 14}
$$

$$
D D=c \Delta V \times(203+D S \times 58-D D \times 42) / m
$$

When Eqs. (1) and (2) were merged, Eqs. (3) and (4) were obtained.

$$
D D=\frac{33.83 \times C / N-29}{m /(c \Delta V)-16}
$$

$$
D S=D D+0.5833 \times C / N-4
$$


where: $\mathrm{C} / \mathrm{N}$ is the mass ratio of $\mathrm{C}$ and $\mathrm{N}$ elements measured from elemental analysis, $m(\mathrm{~g})$ is the sample weight used in the titration experiment, $c(\mathrm{~mol} / \mathrm{L})$ is the concentration of standard $\mathrm{NaOH}$ solution, and $\triangle \mathrm{V}(\mathrm{L})$ is the volumetric difference of standard $\mathrm{NaOH}$ solution between the two peaks in differential titration curve.

The calculated DD and DS values of O-CMCS, F2 F5 according to the Eqs. (3) and (4) mentioned above were presented in Table 1.

\section{Preparation and surface morphology observation of the drug-loading particle}

Exactly $0.2 \mathrm{~g}$ of O-CMCS was completely dissolved in $4.8 \mathrm{~g}$ of deionized water. $2.5 \mathrm{~g}$ of sodium acetylsalicylate solution ( $8 \mathrm{wt} . \%$ ) was added into the O-CMCS solution and stirred continuously. The additional sodium acetylsalicylate solution was prepared by adding $0.8 \mathrm{~g}$ of acetylsalicylic acid into $9.2 \mathrm{~g}$ of NaHCO3 solution (5 wt.\%) with stirring until it was completely dissolved. The drug-loading hydrogel was then prepared by adding $1.5 \mathrm{~mL}$ of glutaraldehyde solution (1 wt.\%) into the above-mixed solution with stirring for $10 \mathrm{~s}$, and then it was allowed to stand overnight. The drug-loading particle was prepared by drying the drug-loading hydrogel in a vacuum oven at $60^{\circ} \mathrm{C}$ for $12 \mathrm{~h}$, and then it was ground to the millimeter scale. The surface morphology of the drug-loading particle was observed by using a field emission scanning electron microscope (JEOL 4000FX, Tokyo, Japan) with ultra-high resolution.

\section{Drug release experimentation}

The simulated gastric juice was prepared by mixing $16.4 \mathrm{~mL}$ of concentrated $\mathrm{HCl}$ and $10 \mathrm{~g}$ of pepsin. Deionized water was used to make up to a volume of $1,000 \mathrm{~mL}$ which replicated a gastric juice at $\mathrm{pH}$ 1.0. On the other hand, the simulated intestinal juice was prepared by mixing potassium dihydrogen phosphate (KH2PO4) solution (6.8 $\mathrm{g}$ of $\mathrm{KH} 2 \mathrm{PO} 4 \mathrm{in} 500 \mathrm{~mL}$ of deionized water and the $\mathrm{pH}$ adjusted to the value of 6.8 by $0.1 \mathrm{~mol} / \mathrm{L}$ of $\mathrm{NaOH}$ solution) and pancreatin solution (10 $\mathrm{g}$ of pancreatin) in a same amount of deionized water that was used to fill up to a volume of $1,000 \mathrm{~mL}$ in the same way as described above.

The drug release experiments were carried out with the rotating basket method as per the Chinese Pharmacopoeia 2010 Edition [13a, 14]. The $0.05 \mathrm{~g}$ of drug-loading particle was loaded in dialysis bag, and then immersed in the $0.5 \mathrm{~L}$ of dissolution medium with the stirring speed of $180 \pm 1 \mathrm{r} / \mathrm{min}$ at $37 \pm$ $0.5^{\circ} \mathrm{C}$. The solution samples were taken out at specified time intervals for the respective measurements of acetylsalicylic acid and salicylic acid concentrations using a UV-VIS spectrophotometer (UV-2501PC, Shimazu, Japan) at 278 and $302 \mathrm{~nm}$ for acetylsalicylic acid and salicylic acid, respectively. UV-VIS spectrophotometer also determined the maximum peak wavelengths and the standard working curves for each acid. The following equation (Eq. (5)) was used to express the cumulative release ratio.

$$
\text { Cumulative release ratio }(\%)=\frac{c m_{2} M_{r} V}{m m_{1}} \times 100 \%
$$


where: $c(\mathrm{~mol} / \mathrm{L})$ is the total concentration of both acetylsalicylic acid and salicylic acid, $m(\mathrm{~g})$ is the weight of drug-loading particle used in the experiment, $m l(0.2 \mathrm{~g})$ is the weight of acetylsalicylic acid loaded in drug-loading particle, $m 2(0.415 \mathrm{~g})$ is the weight of prepared drug-loading particle, $V(\mathrm{~L})$ is the volume of the dissolution medium, and $M r$ is a constant value of 180.16 which represents the molar mass of acetylsalicylic acid.

\section{Statistical analysis}

All experiments were performed in triplicate. All data are presented as mean \pm standard deviation (SD). Statistical significance was determined using Student's t-test. The significance level was set at P < 0.05 .

\section{Results and Discussion}

\section{Surface morphology of the drug-loading particle}

Figure 1 showed surface morphology observed by SEM of the drug-loading particle prepared from F2 (Table 1). The results reveal that the surface morphologies of the blank and all other drug-loading particles developed from this same organic polymer (O-CMCS) with different DD were similar to each other. It turned out that the drug-loading particles expressed an open pore structure, and the interior pore size of these drug-loading particles was measured and bar-lined between 50 and $100 \mu \mathrm{m}$. This structure could raise the contact area between the cross-linked skeleton and dissolution medium, which was beneficial to the formation of a hydrogel.

\section{Drug release performance of the drug-loading particles}

As the acetylsalicylic acid released from drug-loading particles, it may be decomposed into the salicylic acid due to the presence of both acidic solution and pepsin in the simulated gastric juice, both acetylsalicylic acid and salicylic acid were detected at $\mathrm{pH}$ 1.0, and only acetylsalicylic acid was detected at $\mathrm{pH}$ 6.8. The cumulative drug release curves of drug-loading particles based on cross-linked O-CMCS in both simulated gastric and intestinal juices were presented in Fig. 2 and Fig. 3, respectively. As it can be observed, the cumulative drug release ratio of drug-loading particle decreased as the DD of O-CMCS increased, and that of drug-loading particle prepared from O-CMCS with a DD of 51\% was the highest. It could be possibly due to the effect of DD on cross-linking density of the carrier. For all drug-loading particles, the weight ratio of O-CMCS to glutaraldehyde was the same, so the cross-linking density of the cross-linked O-CMCS skeleton equally increased as the DD of O-CMCS (amount of -NH2). Hence, the drug in a corresponding drug-loading particle or hydrogel prepared from O-CMCS with higher DD could not be quickly released.

It is important to emphasize here that the first drug in the drug-loading particle was rapidly released into the simulated gastric juice owe to the liberation of the drug without being encapsulated in the cross- 
linking network. After that, its release rate started to slow down, and its cumulative release ratio slowly began to increase as well. For example, the cumulative drug release ratio of drug-loading particle prepared from O-CMCS with a DD of $51 \%$ in the simulated gastric juice was only around $25.31 \%$ after 720 min. Whereas for all tested drug-loading particles made from the same compound with different DD, both cumulative drug release ratios and release rates in the simulated intestinal juice were all higher than that in the simulated gastric juice. Among them, the highest cumulative drug release ratio of the drugloading particle prepared from $\mathrm{O}-\mathrm{CMCS}$ with a DD of 51\% in the simulated intestinal juice reached up to the extent of $80 \%$ after 720 min (Fig. 3). When the hydrogel was formed by putting a drug-loading particle in an acidic simulated gastric juice, the hydrogen bond density increased owe to the - $\mathrm{COOH}$ of the gel network. Soon after that, it appeared that the drug-loading hydrogel contracted and hindered the internal drug release. In addition, the electrostatic attraction between $-\mathrm{COOH}$ of acetylsalicylic acid/salicylic acid and few residual -NH2 of O-CMCS in the acidic simulated gastric juice could possibly bind both acetylsalicylic acid and salicylic acid, and also dedicated to both low cumulative drug release ratio and low release rate of the drug-loading particle in the simulated gastric juice. However, when the same thing was done in neutral simulated intestinal juice, the $-\mathrm{COOH}$ of gel network was gradually transformed to -COO-. With the repelling action of the negative ions, it was observable that the gel network became loose first, then the drug-loading hydrogel expanded, and finally, the drug was gradually released. Furthermore, the incremental water solubility of acetylsalicylic acid owing to the transformation of $-\mathrm{COOH}$ to $-\mathrm{COO}$ - in neutral simulated intestinal juice accumulated their diffusion and then elevated both cumulative drug release ratio and release rate of the drug-loading particle. The drug release mechanism of the drug-loading hydrogel in simulated gastric/intestinal juices was shown in Fig. 4.

Besides that, the Zero-order, Higuchi, Logistic, and Weibull drug release kinetics models [14a, 15] were fitted to the drug release kinetics of the drug-loading particle prepared from F2 in the simulated intestinal juice. Those results were shown in Table 2. In another development, the comparison of release kinetics curves tailored by these 4 models with the actual practical experiments was given in Fig. 4 . It turned out that both Logistic and Weibull models fitted very well to the experimental results, where their correlation coefficients (R2) were all recorded as 0.99. In addition to that, the release shapes for these 2 models resembled an $S$-type release as expected.

\section{Conclusion}

The drug-controlled release system of a drug-loading hydrogel based on cross-linked O-CMCS was successfully developed. This system proved itself to be reliable and naturally $\mathrm{pH}$ sensitive towards the gastric and intestinal conditions in the humans. Consequently, the drug-loading particle can be applied as a colonic drug too. 
Author statements

Yiguang Wu: Conceptualization, Methodology, Writing - Original Draft, Formal analysis; Muhammad Shahid Riaz Rajoka: Conceptualization, Methodology, Writing - Original Draft, Formal analysis; Jun Zeng: Conceptualization, Methodology, Writing - Original Draft, Formal analysis; Hafiza Mahreen Mehwish: Software, Validation, Data Curation, Writing - Original Draft; Xia Lixin: Funding acquisition, Writing - Review \& Editing, Resources; Wenli Liu: Validation, Formal analysis, Software, Writing Original Draft, Writing - Review \& Editing; Liqing Zhao: Funding acquisition, Project administration, Supervision, Writing - Review \& Editing, Investigation.

\section{Conflict of Interest}

The authors declare that they have no conflicts of interest.

\section{Acknowledgments}

This work was supported by the National Natural Science Foundation of China (21606152 and 31701621), the Natural Science Foundation of Guangdong Province (2016A030313053), the Special Fund for Development of Strategic Emerging Industries in Shenzhen (JCYJ20160520174823939, JCYJ20170817100522830, 20170424181248489), and Fundamental Research Plan (Grant no JCYJ 20170818094217688).

[1] N. Bhattarai, J. Gunn, M. Zhang, Advanced Drug Delivery Reviews 2010, 62, 83-99. aD. Ramyadevi, K. S. Rajan, B. N. Vedhahari, K. Ruckmani, N. Subramanian, Colloids and

\section{References} surfaces. B, Biointerfaces 2016, 146, 260-270; bL. Weng, A. Romanov, J. Rooney, W. Chen, Biomaterials 2008, 29, 3905-3913.

[3] S. B. Makwana, V. A. Patel, S. J. Parmar, Results in Pharma Sciences 2016, 6, 1-6.

[4] aJ. Zhang, N. A. Peppas, Macromolecules 2000, 33, 102-107; bJ. M. Bezemer, R. Radersma, D. W. Grijpma, P. J. Dijkstra, J. Feijen, C. A. van Blitterswijk, Journal of Controlled Release 2000, 64, 179-192.

[5] E. Ruel-Gariépy, J.-C. Leroux, European Journal of Pharmaceutics and Biopharmaceutics 2004, $58,409-426$.

[6] G. Tan, S. Yu, J. Li, W. Pan, International Journal of Biological Macromolecules 2017, 103, 941-947.

[7] aG. Huang, Y. Liu, L. Chen, Drug delivery 2017, 24, 108-113; bJ. Sarvaiya, Y. K. Agrawal, Int J Biol Macromol 2015, 72, 454-465.

[8] aP. Geetha, A. J. Sivaram, R. Jayakumar, C. Gopi Mohan, Carbohydr Polym 2016, 142, 240-249; bT. Jain, S. Kumar, P. K. Dutta, Int J Biol Macromol 2016, 82, 1011-1017. 
268 [9] aA. L. Bukzem, R. Signini, D. M. Dos Santos, L. M. Liao, D. P. Ascheri, Int J Biol Macromol

[10] aY. F. Liu, K. L. Huang, D. M. Peng, P. Ding, G. Y. Li, Int J Biol Macromol 2007, 41, 87-93; bN. A. Gujarathi, B. R. Rane, J. K. Patel, Int J Pharm 2012, 436, 418-425.

[11] C. Liu, Y. Wu, L. Zhao, X. Huang, International Journal of Biological Macromolecules 2015, 78, 189-194.

[12] R. Casado-Arroyo, C. Gargallo, Á. Lanas Arbeloa, Best Practice \& Research Clinical Gastroenterology 2012, 26, 173-184.

[13] aY. J. Wang, E. Assaad, P. Ispas-Szabo, M. A. Mateescu, X. X. Zhu, Int J Pharm 2011, 419, 215221; bX. Huang, Y. Wu, S. Wei, C. Liao, Q. Chen, Int J Biol Macromol 2010, 47, 223-227.

[14] aC. Liu, Y. Wu, L. Zhao, X. Huang, Int J Biol Macromol 2015, 78, 189-194; bL. Pachuau, B. Mazumder, International Journal of Biological Macromolecules 2013, 61, 333-339.

[15] aN. Yuksel, A. E. Kanik, T. Baykara, Int J Pharm 2000, 209, 57-67; bF. O. Costa, J. J. Sousa, A. A. Pais, S. J. Formosinho, Journal of controlled release : official journal of the Controlled Release Society 2003, 89, 199-212. 


\section{Figures}
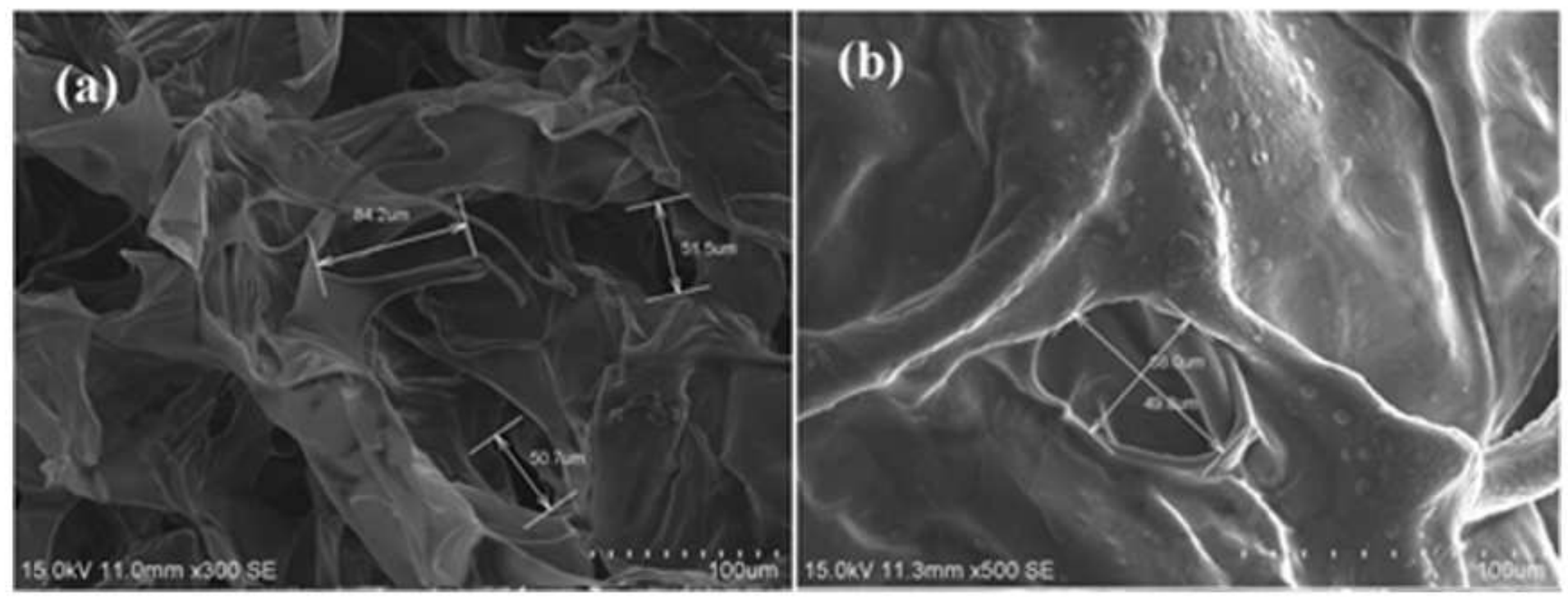

\section{Figure 1}

The surface's SEM photographs of the drug-loading particle prepared from F2

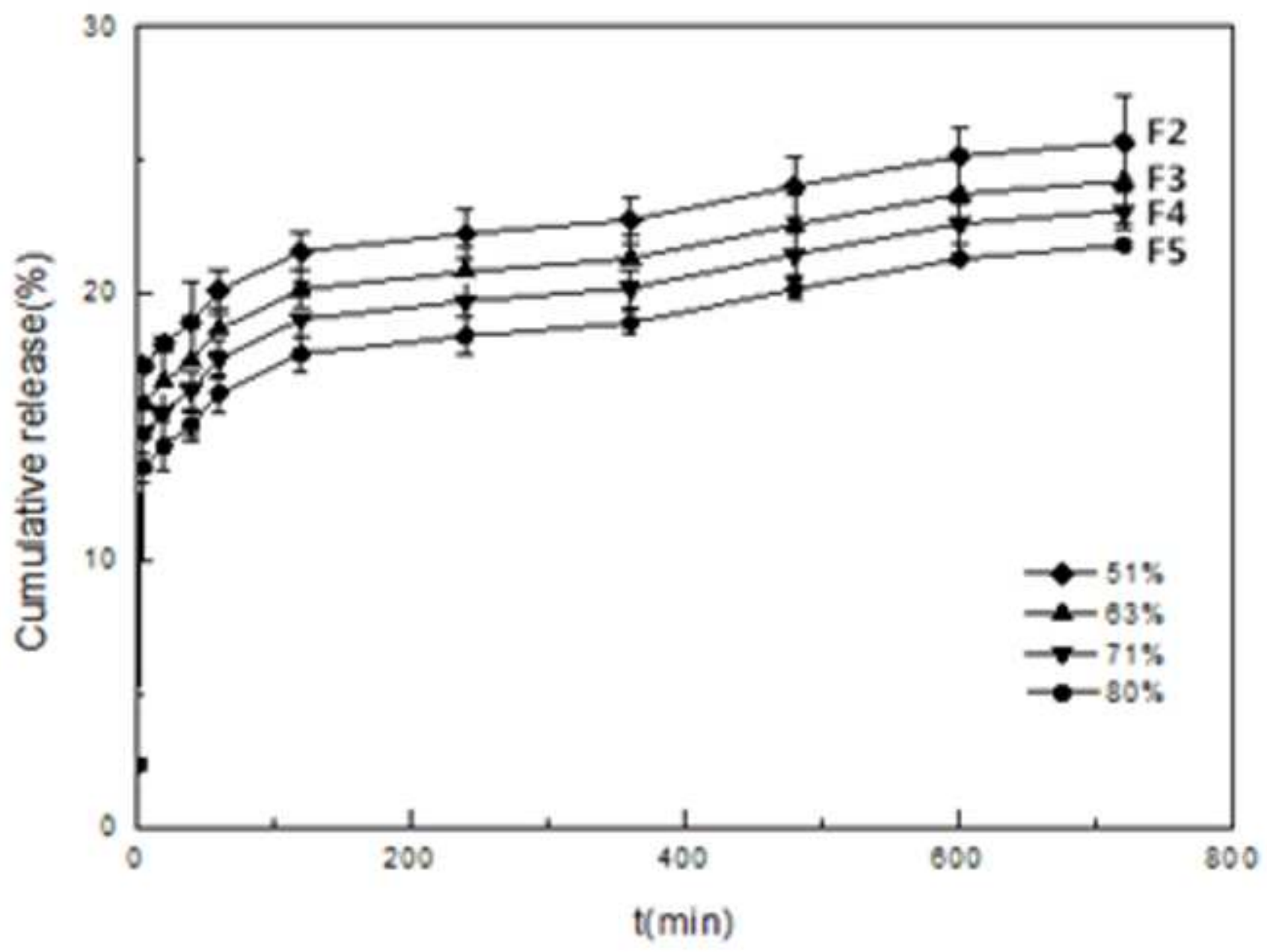


Figure 2

The cumulative drug release curves of the drug-loading particles in simulated gastric juice

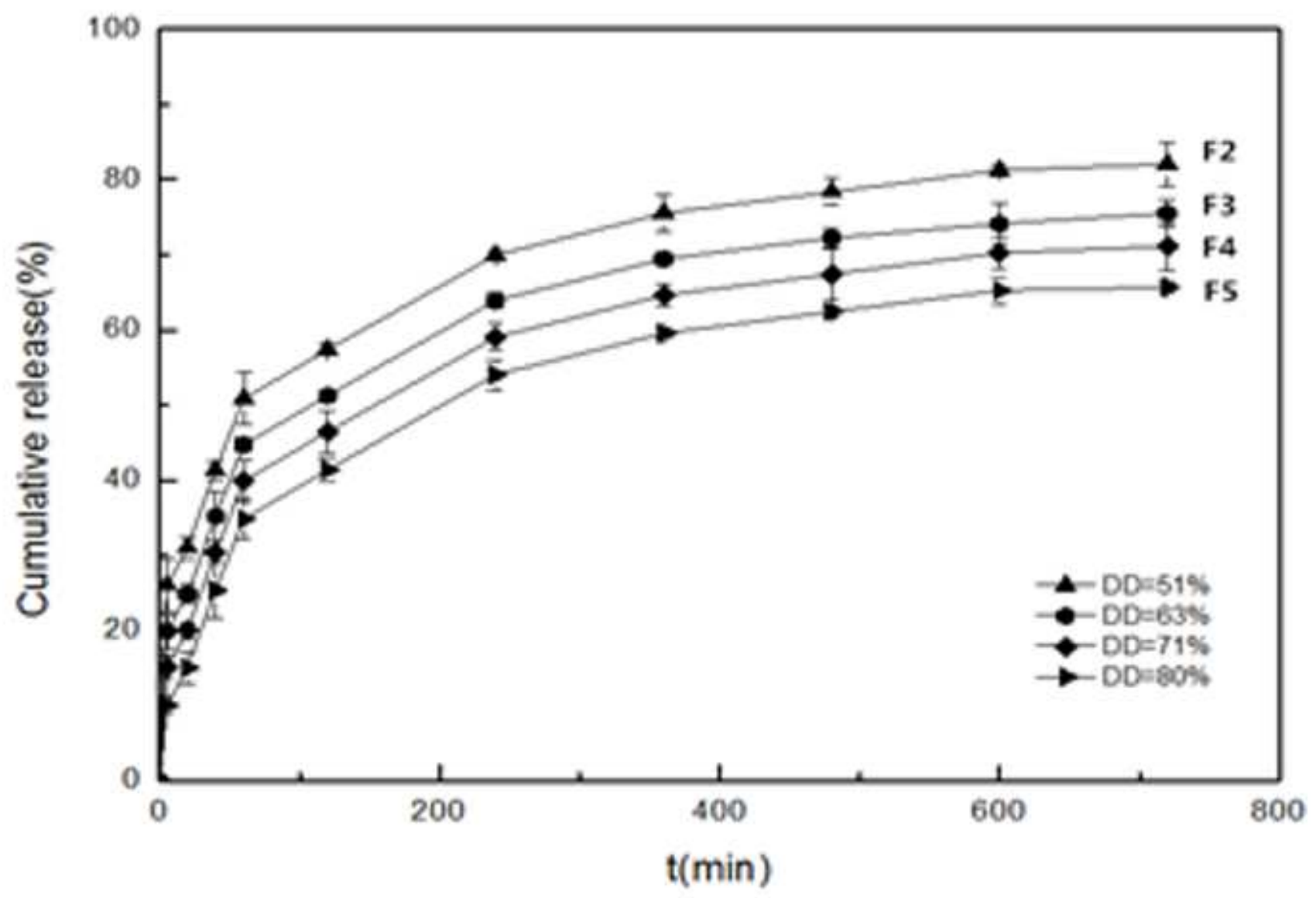

Figure 3

The cumulative drug release curves of the drug-loading particles in simulated intestinal juice 


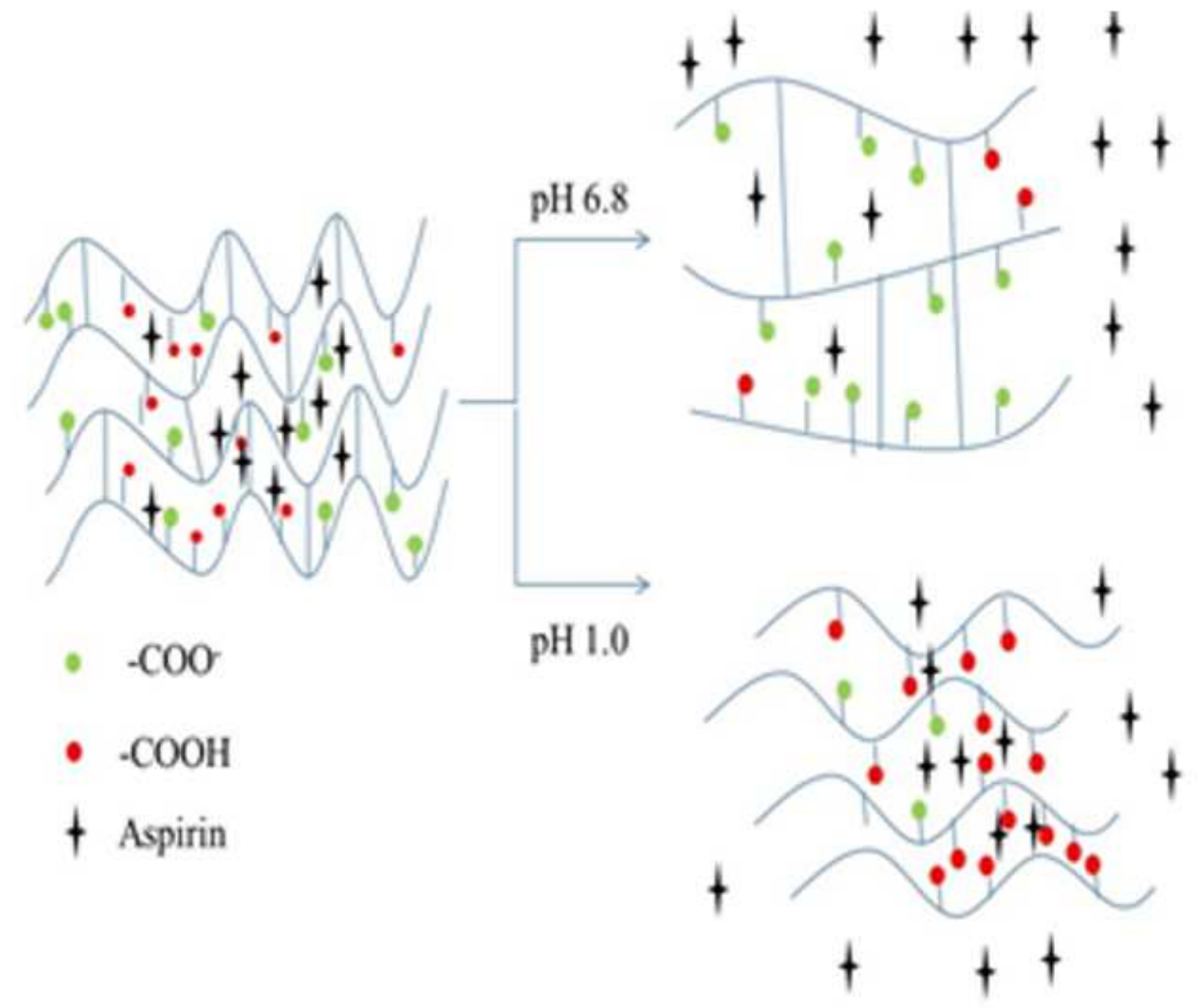

Figure 4

The drug release mechanism of the drug-loading hydrogel in simulated gastric/intestinal juices 


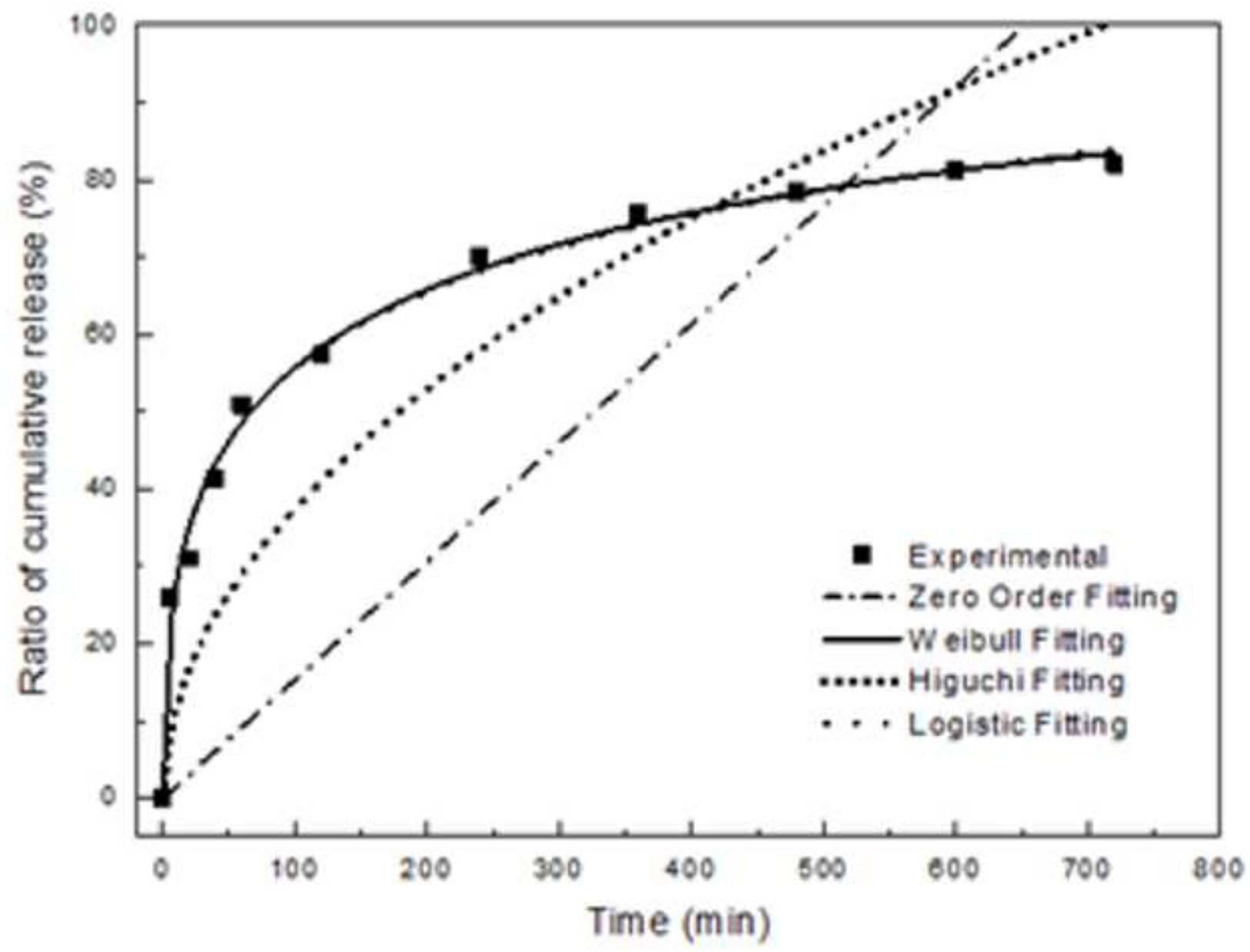

Figure 5

The comparison of release kinetics curves fitted by the four models with the experimental

\section{Supplementary Files}

This is a list of supplementary files associated with this preprint. Click to download.

- Table.docx 